\title{
Overlooked Trustworthiness of Explainability in Medical Al
}

\author{
Jiajin Zhang, \#, Hanqing Chao ${ }^{1, \#, ~ M a n n u d e e p ~ K . ~ K a l r a ~}{ }^{2}$, Ge Wang ${ }^{1}$, Pingkun Yan ${ }^{1, *}$ \\ 1. Department of Biomedical Engineering, Center for Biotechnology and Interdisciplinary Studies, \\ Rensselaer Polytechnic Institute, Troy, NY, USA. \\ 2. Department of Radiology, Massachusetts General Hospital, \\ Harvard Medical School, Boston, MA, USA. \\ \# Equal contributions, * Corresponding author (yanp2@rpi.edu)
}

\begin{abstract}
While various methods have been proposed to explain Al models, the trustworthiness of the generated explanation received little examination. This paper reveals that such explanations could be vulnerable to subtle perturbations on the input and generate misleading results. On the public CheXpert dataset, we demonstrate that specially designed adversarial perturbations can easily tamper saliency maps towards the desired explanations while preserving the original model predictions. Al researchers, practitioners, and authoritative agencies in the medical domain should use caution when explaining Al models because such an explanation could be irrelevant, misleading, and even adversarially manipulated without changing the model output. Al researchers, practitioners, and authoritative agencies in the medical domain should use caution when explaining Al models because such an explanation could be irrelevant, misleading, and even adversarially manipulated without changing the model output.
\end{abstract}

\section{Introduction}

Explainability is a pillar in supporting applications of artificial intelligence and machine learning $(\mathrm{Al} / \mathrm{ML})$ in medicine ${ }^{1}$. Understanding the how and why $\mathrm{Al}$ models make particular decisions is critical for building trust in any Al-driven application². There is a large body of research papers on methods revealing how Al works ${ }^{3,4}$. The Food and Drug Administration (FDA) is the federal agency responsible for clearing medical Al devices for clinical use in the United States. FDA stresses trustworthiness as a key factor for adopting and evolving medical $\mathrm{Al} / \mathrm{ML}$ devices, a viewpoint endorsed by more than 40 other countries ${ }^{5}$. While the research community appreciates the development of explainable $\mathrm{Al}(\mathrm{XAI})$, we often focus on how to explain an Al model but have not answered an important question - can the explainability be trusted? The trustworthiness of Albased saliency methods has been overlooked. This has left potential risks to Al-based applications in the medical field.

To understand the extent of these concerns, here we demonstrate worrisome phenomena with saliency maps in disease diagnosis from medical images through both case studies and statistical analysis. Saliency maps, also commonly referred to as heat maps, are the most commonly used method for Al explainability ${ }^{6}$. The research community is increasingly aware that the output of an Al model could abruptly change with subtle perturbations to the input, especially adversarial attacks $^{7}$. Little research has been done to understand the impact of tampered inputs on the explainability of $\mathrm{Al}^{8}$. It is therefore imperative to pay attention to the trustworthiness of explainability in medical Al.

\section{Methods}

\subsection{Saliency Maps Can Be Misleading}


medRxiv preprint doi: https://doi.org/10.1101/2021.12.23.21268289; this version posted December 24, 2021. The copyright holder for this preprint (which was not certified by peer review) is the author/funder, who has granted medRxiv a license to display the preprint in perpetuity.

It is made available under a CC-BY-NC-ND 4.0 International license .

We compiled a representative list of medical Al papers utilizing the various saliency map generation methods (referred to as saliency methods for conciseness) for their explainability (https://github.com/DIAL-RPI/Trustworthiness-of-Medical-XAl). In this list, seven most commonly used saliency methods, including Vanilla $\operatorname{Grad}^{9}$, $\operatorname{Grad}^{\mathrm{x}} \operatorname{Image}^{10}$, $\operatorname{GradCAM}^{6}$, Guided-GradCAM ${ }^{6}$, $\mathrm{IG}^{11}, \mathrm{SG}^{12}$ and XRAI ${ }^{13}$, were selected with applications in medical Al applications. We used a public chest radiograph dataset, CheXpert, ${ }^{14}$ and a widely used baseline model, DenseNet ${ }^{15}$, to demonstrate potential problems with saliency maps. Technical details of model training are publicly available at (https://github.com/DIAL-RPI/Trustworthiness-of-Medical-XAl). We evaluated the model performance by the area under the receiver operating characteristic curve (AUC). Following the original work of CheXpert ${ }^{14}$, we focused on five observations: (a) Atelectasis, (b) Cardiomegaly, (c) Consolidation, (d) Edema, and (e) Pleural Effusion. Our DenseNet achieved a mean AUC of 0.895 , slightly surpassing the performance presented in the original paper ${ }^{14}$.
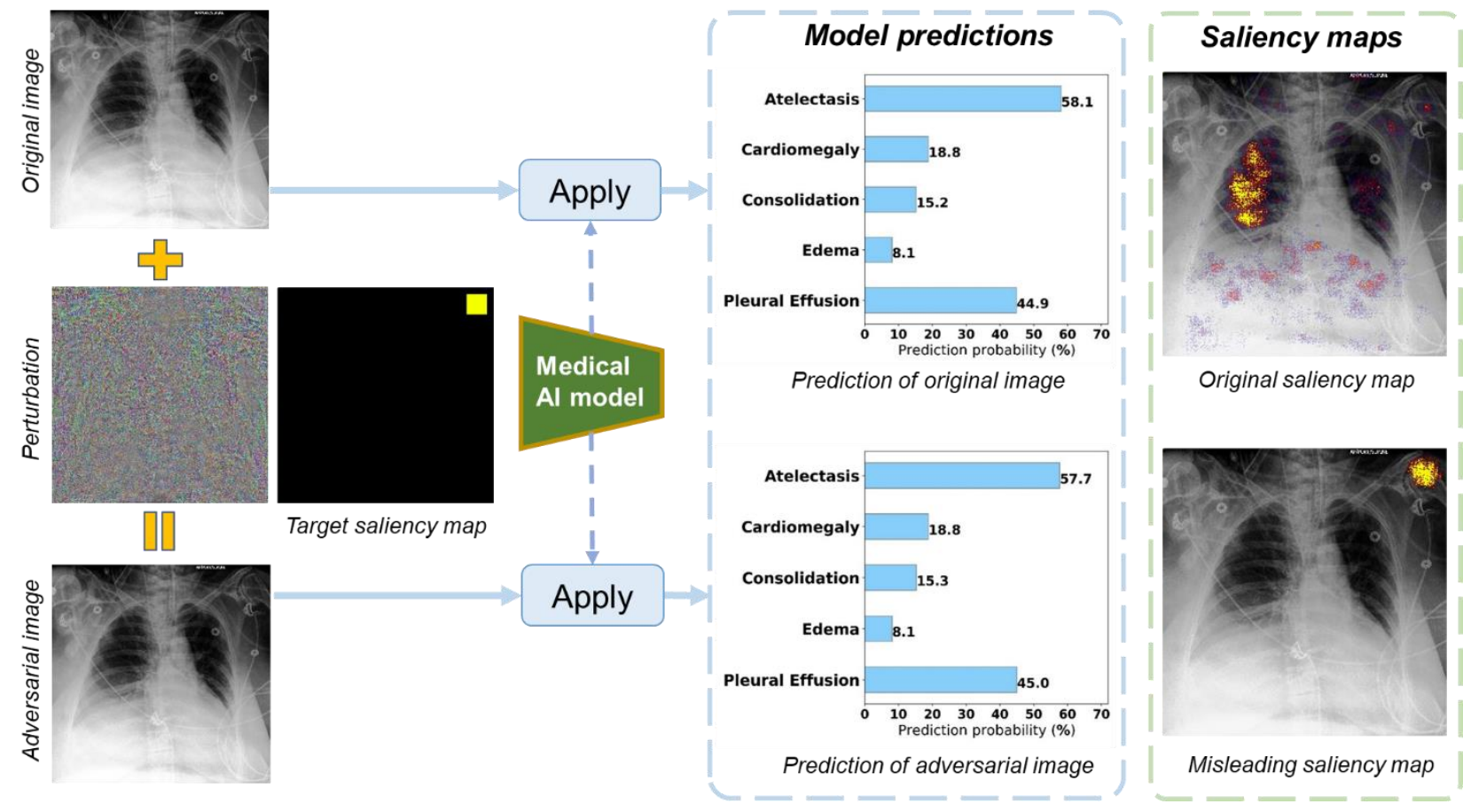

Figure 1. Our study illustrated by an example, where an adversarial image with humanimperceptible tampers the saliency map without changing the model predictions. The data and model used in this example are available at (https://github.com/DIAL-RPI/Trustworthiness-ofMedical-XAI).

The objective of our study is to demonstrate that saliency maps of an Al model can be easily manipulated by applying human imperceptible perturbations to an input image without noticeable change of the model output. Fig. 1 illustrates our study. Given an original input image and the trained Al model, an adversarial image can be generated by adding human-imperceptible perturbations to the original image. More specifically, the adversarial counterpart can be computed by minimizing the loss

$L\left(x_{\text {adv }}\right)=\alpha_{1}\left\|m\left(x_{\text {adv }}\right)-m_{\text {target }}||^{2}+\alpha_{2}\right\| f\left(x_{\text {adv }}\right)-f\left(x_{\text {origin }}\right)\left\|^{2}+\right\| x_{\text {adv }}-x_{\text {origin }} \|^{2}$,

where $m(*)$ represents a certain saliency method, $f(*)$ denotes the Al model, the hyperparameter $\alpha_{1}$ and $\alpha_{2}$ were empirically set to $10^{14}$ and $10^{3}$, respectively, in our experiment. 
By minimizing the loss through manipulating an adversarial image $x_{a d v}$, we can have the associated saliency map $m\left(x_{a d v}\right)$ very close to an arbitrarily designed target saliency map $m_{\text {target }}$. At the same time the adversarial image and the model output only deviate slightly from the original image and the corresponding output, respectively. As shown in Fig. 1, when the original image and its adversarial counterpart are fed into the same medical Al model, the outputs of the Al model are in excellent agreement. However, the saliency map of the adversarial image has been tampered towards the intentionally designed pattern. In this case, we specifically made the saliency region irrelevant to the model prediction, which is located on the top right corner of the image. This finding is concerning as it shows not only the saliency map can be completely irrelevant but also such a map can be purposely manipulated to a desired target region. This safety issue significantly reduces the trustworthiness of the explainability of saliency maps.

\subsection{The Concern is Universal}

Next, we underline that this concern is not only universal to the commonly used saliency methods summarized above but also the adversarial images are transferrable across different saliency methods. Specifically, we demonstrate the misleading effects on all the seven representative saliency methods in Fig. 2. The first row of Fig. 2 shows the saliency maps generated on the original input image. The highlighted regions correspond to the observation of atelectasis annotated for this frontal view X-ray image. The adversarial images can be generated for each method following our method described above, which are publicly available at (https://github.com/DIAL-RPI/Trustworthiness-of-Medical-XAl/samples). These adversarial images did not change the model predictions but did falsify saliency maps consistently as shown in the third row of Fig. 2. For all the seven methods, the adversarial images succeeded in shifting the saliency areas to the targeted top right corner of the image. In other words, even if multiple explanation methods produce consistent results, they may still be misleading. 


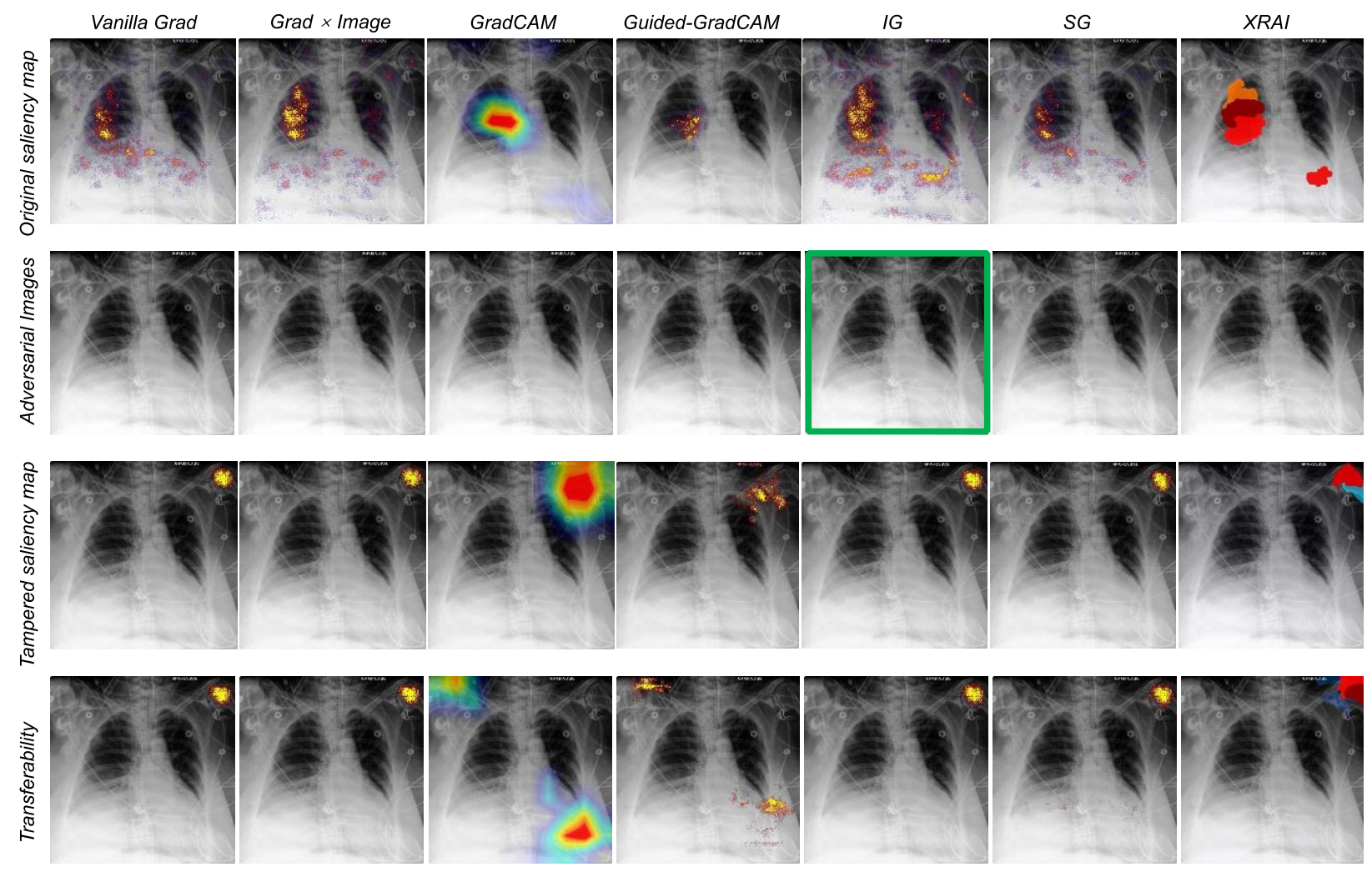

Figure 2. Collusion of explanation methods. First row: saliency maps generated using different methods on the same original input image; Second row: generated adversarial images for each saliency visualization method. Third row: tampered saliency maps using the above adversarial images; Last row: saliency maps generated on the adversarial image highlighted by the green box in the second row.

We further examined the existence of universal adversarial images by evaluating the transferability of the adversarial images across different XAI methods. We used the adversarial image generated for attacking IG as input and produced saliency maps using other methods. The last row of Fig. 3 shows that the adversarial image towards IG can be well transferred to attack the other methods by shifting the attention areas. For majority of the methods, the attention area was moved to the targeted top-right corner of the image. More cases studies can be found at (https://github.com/DIAL-RPI/Trustworthiness-of-Medical-XAl).

\subsection{Ensuring Al Model Prediction Consistence is Not Enough}

We quantitatively evaluated the image similarities and saliency map similarities as well as the model prediction consistence on 200 chest radiographs from the CheXpert validation set. The similarities were assessed in terms of the structural similarity index measure (SSIM) ${ }^{16}$. The results

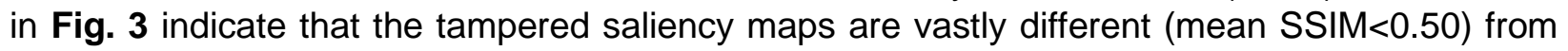
the corresponding maps for the original images, while the adversarial images are remarkably close to the original images (mean SSIM>0.98). This indicates that the perturbations added to the adversarial images may be hardly noticeable by humans but they could cause significant changes to the saliency maps. Additional evaluation metrics including Mean-Square Error (MSE) and Pearson Correlation Coefficient (PCC) were also used to provide the same conclusion. The results are available at (https://github.com/DIAL-RPI/Trustworthiness-of-Medical-XAl). 
medRxiv preprint doi: https://doi.org/10.1101/2021.12.23.21268289; this version posted December 24, 2021. The copyright holder for this preprint (which was not certified by peer review) is the author/funder, who has granted medRxiv a license to display the preprint in perpetuity.

It is made available under a CC-BY-NC-ND 4.0 International license .

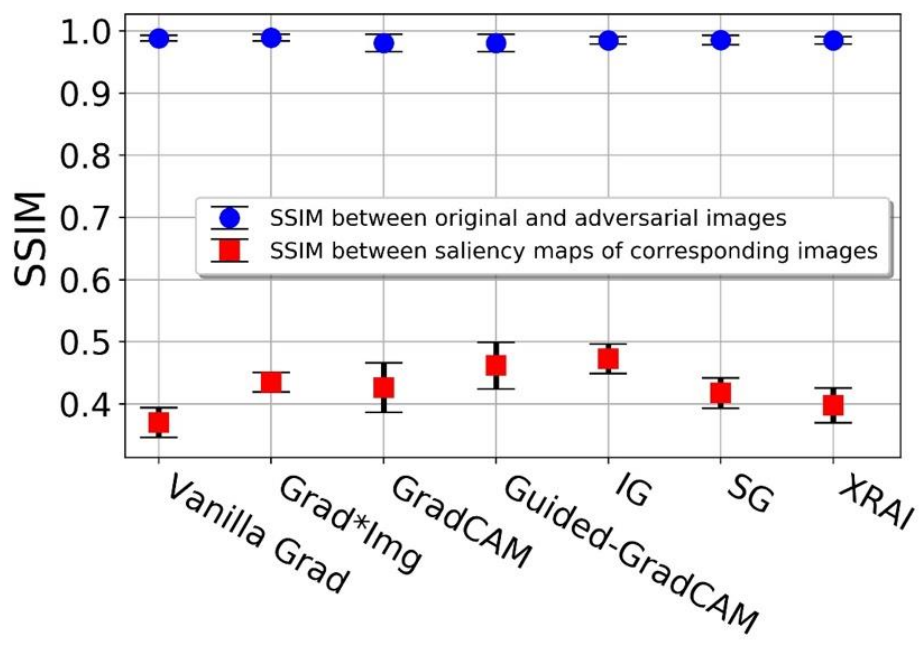

Figure 3. Comparation between original and adversarial results. Blue box plots: similarities between the original images and the adversarial images; and red box plots: similarities between the saliency maps for the original and adversarial inputs. The dots and squares in the plots denote the mean values, and the bars indicate the standard deviations.

In addition, we evaluated the effect of the adversarial images on the model performance measured by AUC, prediction consistency assessed by AUC change percentage relative to the original value of 0.895 , and unsuccessful attack ratio. An attack is considered as failed if the SSIM between the tampered saliency map and the target map is below 0.6. The results in Table 1 show that the Al model performed consistently under the original input images and adversarial images, with the performance change less than $2.5 \%$. At the same time, the saliency maps were successfully manipulated towards the target with the unsuccessful attack rates all below $10 \%$ for all the XAl methods. These results demonstrate that model saliency maps as an explainability approaches can be purposely manipulated even the predictions remain consistent. That is, the correctness of the model predictions may not support the validity of explanations to the model.

Table 1. Saliency maps are successfully manipulated with only slight influence to the model performance.

\begin{tabular}{|c|c|c|c|c|c|c|c|}
\hline Methods & $\begin{array}{c}\text { Vanilla } \\
\text { Grad }\end{array}$ & $\begin{array}{c}\text { Vanilla } \\
\text { GradxImage }\end{array}$ & GradCAM & $\begin{array}{c}\text { Guided } \\
\text { GradCAM }\end{array}$ & IG & SG & XRAI \\
\hline $\begin{array}{c}\text { AUC } \\
\text { (change \%) }\end{array}$ & $\begin{array}{c}0.888 \\
(-0.7 \%)\end{array}$ & $\begin{array}{c}0.882 \\
(-1.3 \%)\end{array}$ & $\begin{array}{c}0.870 \\
(-2.5 \%)\end{array}$ & $\begin{array}{c}0.870 \\
(-2.5 \%)\end{array}$ & $\begin{array}{c}0.882 \\
(-1.3 \%)\end{array}$ & $\begin{array}{c}0.887 \\
(-0.8 \%)\end{array}$ & $\begin{array}{c}0.882 \\
(-1.3 \%)\end{array}$ \\
\hline $\begin{array}{c}\text { Unsuccessful } \\
\text { attack ratio }\end{array}$ & $1.3 \%$ & $1.0 \%$ & $0.2 \%$ & $0.1 \%$ & $9.3 \%$ & $5.2 \%$ & $0.2 \%$ \\
\hline
\end{tabular}

\section{Discussions and Conclusions}

The input images in our experiment were adversarially perturbed to reveal severe safety issues of the existing XAI methods. Such perturbations could appear naturally in medical imaging due to numerous variations in commercial vendors, acquisition parameters, image reconstruction algorithms, patient characteristics, and so on. Also, malicious human hackers in the healthcare domain were reported before ${ }^{17}$, and their persistent existence should be a valid assumption. Given the limited evaluation of findings with most FDA cleared AI models, the reported problems 
medRxiv preprint doi: https://doi.org/10.1101/2021.12.23.21268289; this version posted December 24, 2021. The copyright holder for this preprint (which was not certified by peer review) is the author/funder, who has granted medRxiv a license to display the preprint in perpetuity.

It is made available under a CC-BY-NC-ND 4.0 International license .

could arise in presence of distracting or mimicking findings to the intended target for Al algorithms. Therefore, the findings in this paper suggest that Al researchers, practitioners and authoritative agencies in the medical domain must use caution when explaining Al models in their applications with saliency maps, and perhaps other types of explanation methods as well. Solid qualityassurance measures are urgently needed to increase and validate the trustworthiness of Al explainability, especially in the medical field.

\section{References}

1. Kundu, S. Al in medicine must be explainable. Nat Med 27, 1328-1328 (2021).

2. Ghassemi, M., Oakden-Rayner, L. \& Beam, A. L. The false hope of current approaches to explainable artificial intelligence in health care. The Lancet Digital Health 3, e745-e750 (2021).

3. Tjoa, E. \& Guan, C. A Survey on Explainable Artificial Intelligence (XAI): Toward Medical XAI. IEEE Trans. Neural Netw. Learning Syst. 32, 4793-4813 (2021).

4. Arun, N. et al. Assessing the (Un)Trustworthiness of Saliency Maps for Localizing Abnormalities in Medical Imaging. Radiology: Artificial Intelligence (2021) doi:10.1148/ryai.2021200267.

5. U.S. Food \& Drug Administration Center for Devices and Radiological Health. Artificial Intelligence (AI) and Machine Learning (ML) in Medical Devices. (2020).

6. Selvaraju, R. R. et al. Grad-cam: Visual explanations from deep networks via gradient-based localization. in Proceedings of the IEEE international conference on computer vision (ICCV) 618-626 (2017).

7. Zhou, Q. et al. A machine and human reader study on Al diagnosis model safety under attacks of adversarial images. Nat Commun 12, 7281 (2021).

8. Dombrowski, A.-K. et al. Explanations can be manipulated and geometry is to blame. Advances in Neural Information Processing Systems 32, 13589-13600 (2019).

9. Simonyan, K., Vedaldi, A. \& Zisserman, A. Deep Inside Convolutional Networks: Visualising Image Classification Models and Saliency Maps. arXiv:1312.6034 [cs] (2014). 
10. Shrikumar, A., Greenside, P., Shcherbina, A. \& Kundaje, A. Not Just a Black Box:

Learning Important Features Through Propagating Activation Differences. arXiv:1605.01713 [cs] (2017).

11. Sundararajan, M., Taly, A. \& Yan, Q. Axiomatic Attribution for Deep Networks. arXiv:1703.01365 [cs] (2017).

12. Smilkov, D., Thorat, N., Kim, B., Viégas, F. \& Wattenberg, M. SmoothGrad: removing noise by adding noise. arXiv:1706.03825 [cs, stat] (2017).

13. Kapishnikov, A., Bolukbasi, T., Viegas, F. \& Terry, M. XRAI: Better Attributions Through Regions. in 2019 IEEE/CVF International Conference on Computer Vision (ICCV) 4947-4956 (IEEE, 2019). doi:10.1109/ICCV.2019.00505.

14. Irvin, J. et al. CheXpert: A Large Chest Radiograph Dataset with Uncertainty Labels and Expert Comparison. AAA/ 33, 590-597 (2019).

15. landola, F. et al. DenseNet: Implementing Efficient ConvNet Descriptor Pyramids. arXiv:1404.1869 [cs] (2014).

16. Wang, Z., Bovik, A. C., Sheikh, H. R. \& Simoncelli, E. P. Image Quality Assessment: From Error Visibility to Structural Similarity. IEEE Trans. on Image Process. 13, 600-612 (2004).

17. Eichelberg, M., Kleber, K. \& Kämmerer, M. Cybersecurity Challenges for PACS and Medical Imaging. Academic Radiology 27, 1126-1139 (2020). 